New Concepts in Imaging: Optical and Statistical Models

D. Mary, C. Theys and C. Aime (eds)

EAS Publications Series, 59 (2013) 25-36

\title{
OPTICAL LONG BASELINE INTERFEROMETRY: EXAMPLES FROM VEGA/CHARA
}

\author{
D. Mourard ${ }^{1}$
}

\begin{abstract}
In this paper I review some of the fundamental aspects of optical long baseline interferometry. I present the principles of image formation, the main difficulties and the ways that have been opened for high angular resolution imaging. I also review some of the recent aspects of the science program developed on the VEGA/CHARA interferometer.
\end{abstract}

\section{Introduction}

Astrophysics is based on observations and physical analysis. From the point of view of observations, this science has mainly been developed through the progresses in the techniques of imaging, astrometry, photometry, spectroscopy and polarimetry. However, through these techniques, objects are almost always considered as pointlike source and no information is obtained on their brightness distribution. This is of course due to the diffraction principle, the limited size of the collecting optics used in telescopes and the very small apparent angular sizes of these objects.

In 1974, A. Labeyrie succeeded for the first time to obtain interference fringes on a stellar source with two separate telescopes. This achievement opened the road for the modern development of optical interferometry and allowed to give access to astrophysics at very high angular resolution. Today, the situation is dominated by a few facilities: mainly the VLTI (Glindemann et al. 2004), KECK (Colavita et al. 2006) and the CHARA array (Ten Brummelaar et al. 2005), allowing combination of 4 to 6 telescopes from the visible to the thermal infrared domain. With almost 50 scientific papers per year, the progression of the astrophysical impact of long baseline optical interferometry is almost following, with a time shift of 30 years, the development of radio interferometry.

The main scientific domains of modern optical long baseline interferometry are the study of brightness distribution of compact objects such as stellar surfaces,

${ }^{1}$ Laboratoire Lagrange, UMR 7293, UNS-CNRS-OCA, Boulevard de l'Observatoire, BP. 4229, 06304 Nice Cedex 4, France 


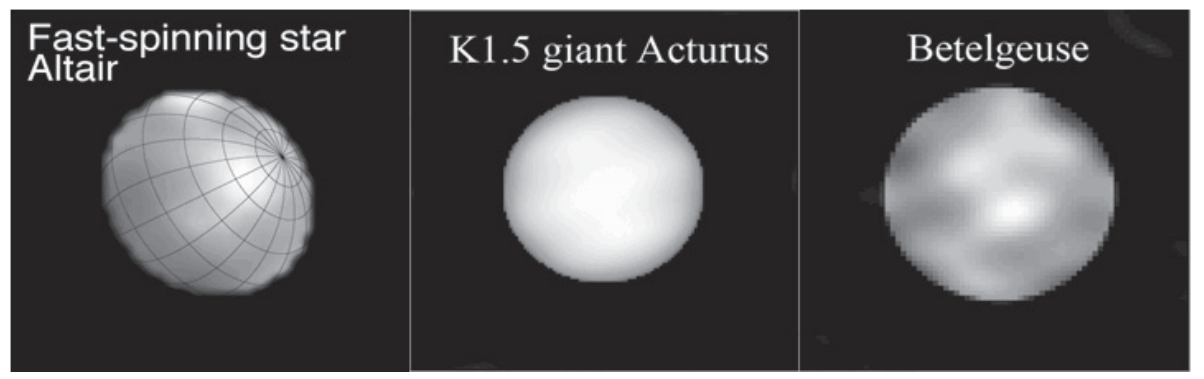

Fig. 1. Recent examples of stellar surface images obtained by optical long baseline interferometers. Left: Monnier et al. (2007). Middle: Lacour et al. (2008). Right: Haubois et al. (2008).

cores of young stellar object environments or central regions of active galactic nuclei. These studies require high resolution in the space, time and spectral domains for a correct understanding of the physical processes in action in these objects. As an imaging technique, optical long baseline interferometry performance is highly related to the properties of the field of view and of the transfer function. Recent advances by different groups in the world have lead to the first images of stellar surfaces (see Fig. 1).

Although these first images show remarkable progresses in that field, it is clear however that more technical work is needed to improve the impact of long baseline interferometry. The main issue is certainly the need for angular resolution that requires long baseline $(B>100 \mathrm{~m})$ and short wavelengths $(\lambda<1 \mu \mathrm{m})$ to reach resolution lower than the millisecond of degree needed to resolve details on the surface of stars. Also a much higher dynamic range in the images will be necessary which corresponds in fact to a better sensitivity and an improved signal to noise ratio in the raw measurements. This last point is of course related to the improvement of the limiting magnitude of the technique which is absolutely mandatory for large programs in the extragalactic domain.

In Section 2, I review some of the general principles of optical long baseline interferometry. In Section 3, I will show that optical interferometry is mainly an imaging technique and will detail the most important aspects of this point of view. I present in Section 4, the main limitations encountered and the way optical long baseline interferometry is currently implemented as an observing technique. After a rapid presentation of the CHARA Array and the VEGA instrument in Section 5, I will present recent results obtained by the VEGA group in Section 6 .

\section{Principles of optical interferometry}

This section does not intend to present a complete and rigorous demonstration of the principles of optical interferometry. This is of course out of the scope of this paper and the reader could refer to the excellent book of Goodman (2000) as well 
as to many other reviews. The idea is to present by different point of views the principle of the physical properties of long baseline interferometry.

\subsection{Coherence of the stellar wave}

If we consider a star located at infinity and presenting an angular diameter $\theta$, this object defines a solid angle $\Omega$ defined by:

$$
\Omega=\pi\left(\frac{\theta}{2}\right)^{2} .
$$

We consider a screen of radius $r$ receiving the stellar wave. This screen has a surface $S=\pi r^{2}$. This defines a beam etendue $\epsilon$ that can be written as:

$$
\epsilon=S \Omega=\pi^{2} r^{2}\left(\frac{\theta}{2}\right)^{2} .
$$

The principle of coherence, as defined by Goodman in his book, indicates that we can consider the wave as coherent if $\epsilon<\lambda^{2}$. This defines a so-called radius of coherence $r_{c}$ :

$$
r_{c}=\frac{\lambda}{\pi\left(\frac{\theta}{2}\right)} .
$$

One can note that in the case of a star with an angular diameter $\theta=10$ mas and at a wavelength $\lambda=1 \mu \mathrm{m}$, this leads to a value of $r_{c} \simeq 13 \mathrm{~m}$. We thus understand that it exists a relation between the coherence of the wave and the angular diameter of the star. The coherence of the electromagnetic wave $\psi$ could be determined by the computation of the complex degree of mutual coherence $\left(\Gamma_{12}\right)$ between two points of the collecting screen separated by a distance B.

$$
\Gamma_{12}=\frac{\left|\psi_{1} \psi_{2}{ }^{*}\right|}{\sqrt{\left|\psi_{1}\right|^{2}\left|\psi_{2}\right|^{2}}} .
$$

By using the Van-Cittert Zernike theorem and the notation Õ for the Fourier Transform of the star brightness distribution, we can write the following relation:

$$
\Gamma_{12}=\frac{\tilde{O}\left(\frac{B}{\lambda}\right)}{\tilde{O}(0)} .
$$

Considering the star as a uniform disk, we finally obtain:

$$
\Gamma_{12}=\left|\frac{2 J_{1}(\pi B \theta / \lambda)}{\pi B \theta / \lambda)}\right| .
$$

The definition of coherence by Goodman corresponds to the case where $\Gamma_{12}=0.5$ which corresponds to $\pi B \theta / \lambda=2$ and thus to $B=r_{c}=\frac{\lambda}{\pi\left(\frac{\theta}{2}\right)}$, which is an other way of defining the coherence $\left(\epsilon<\lambda^{2}\right)$. 
This simple calculation shows that the coherence of the electromagnetic wave of stellar sources could be measured through a spatial sampling if one can access to very long baselines (B larger than $100 \mathrm{~m}$ typically). In this paper we only consider the case of direct interferometry in the optical domain, which means that we use detectors sensitive to the intensity of the electromagnetic wave and that we record the intensity resulting from the coherent addition of the complex waves. Coming back to the simple case of an instrumental setup dedicated to the measurement of the complex degree of mutual coherence, a practical implementation of this experiment is thus to consider the coherent addition of the two complex waves collected at point 1 and 2 with a phase shift on the second one dedicated to the necessary adjustment of the optical path between the two wave collectors. Thus we obtain the total intensity $I$ as:

$$
\begin{aligned}
& I=\left|\psi_{1}+\psi_{2} e^{i \phi}\right|, \\
& I={\psi_{1}}^{2}+{\psi_{2}}^{2}+2 \psi_{1} \psi_{2}{ }^{*} \cos (\phi) .
\end{aligned}
$$

Denoting $I_{i}$ the intensity of the wave at point i, we finally obtain:

$$
I=\left(I_{1}+I_{2}\right)\left(1+\frac{2 \sqrt{I_{1} I_{2}}}{I_{1}+I_{2}} * \frac{\psi_{1} \psi_{2}^{*}}{\sqrt{\left|\psi_{1}\right|^{2}\left|\psi_{2}\right|^{2}}} * \cos (\phi)\right)
$$

The term with the cosinus function represents, if one introduces variations of $\phi$ either by temporal or spatial sampling, a modulation in the measured intensity, which is also called interference fringes. The amplitude of the modulation is defined by the factor in front of the cosinus. It contains two parts: the photometric one $\left(\frac{2 \sqrt{I_{1} I_{2}}}{I_{1}+I_{2}}\right)$ and the coherence one $\left(\frac{\psi_{1} \psi_{2}{ }^{*}}{\sqrt{\left|\psi_{1}\right|^{2}\left|\psi_{2}\right|^{2}}}\right)$ where we recognizes $\Gamma_{12}$ the complex degree of mutual coherence of the two collected waves.

As a conclusion of this section, we see that we have indeed a way to measure complex degrees of mutual coherence of stellar waves allowing us to sample the Fourier transform of brightness distributions at very high spatial frequencies. We will see in Section 4 how this method is now implemented in reality but before coming into the instrumental part of this technique an other point of view is also very important for a correct understanding of this observing technique.

\section{Interferometry and images}

Astronomers have developed optical interferometry in order to improve the resolving power of the telescopes. Indeed image formation in a telescope is a standard diffraction problem and it is known for a long time that an image is obtained as the convolution of the brightness distribution of the source by the point spread function of the optical device. When this convolution relation is translated into the Fourier domain, it shows that the spatial frequency spectrum of an image is the spatial frequency spectrum of the object filtered by the optical transfer function 
(OTF) of the optical device. Thanks to the diffraction principle, it could be easily shown that the modulus of the optical transfer function, called the modulation transfer function (MTF), is obtained as the autocorrelation of the pupil function, defining the entrance plane of the optical device.

In the case of a monolithic telescope of diameter D, the OTF acts as a low pass filter transmitting the spatial frequencies of the object brightness distribution up to $D / \lambda$. This corresponds to what is usually called the diffraction limit $\lambda / D$ of the telescope. We do not consider here the perturbations induced by the atmosphere and we just consider the ideal case of a perfect optical instrument.

In the case of an interferometer with two telescopes of diameter D and separated by a vector $\vec{B}$, the support of the OTF (also called the (u,v) plane), is made of a low frequency peak of extent $\pm D / \lambda$ and two high frequency peaks of extent $\pm D / \lambda$ and located at $\pm \vec{B} / \lambda$. The interferometer acts thus as a high frequency band pass filter, allowing to reach information at a resolution of $\lambda /|\vec{B}|$.

In the general case, the $(\mathrm{u}, \mathrm{v})$ plane (support of the OTF) is a function of the input baselines, of the latitude of the observatory, of the target coordinates, of the wavelength and of the time (because of the earth rotation). The $(u, v)$ plane coverage defines the sampling of Fourier transform of the object brightness distribution.

The properties of the image obtained directly at the focus of an interferometer clearly depend on the $(\mathrm{u}, \mathrm{v})$ plane coverage but it can also be shown (Labeyrie 1996) that the beam combination scheme plays also an important role in that domain. I refer the reader to the important papers published in that domain (see Labeyrie et al., these proceedings). As an illustration we present in Figure 2, some examples of $(\mathrm{u}, \mathrm{v})$ plane coverage and point spread function for different kind of optical interferometers.

Currently, no interferometer is working in a direct imaging scheme except maybe the Large Binocular Telescope. The limitations of coherence for ground based projects in the optical domain are clearly difficult to overcome. Progresses are being made in that direction but for the moment, imaging at high angular resolution, is not working directly at the focus of the interferometer. Instead, astronomers are using the $(\mathrm{u}, \mathrm{v})$ plane coverage to sample the Fourier transform of the brightness distribution and then to reconstruct images. This method has made great progresses in the recent years as shown in Figure 1. The quality of the reconstructed images highly depends of the $(\mathrm{u}, \mathrm{v})$ plane coverage and of the a priori information (regularization constraints) introduced in the reconstruction algorithm. I do not intend to describe this method in the present paper and I refer the reader to the chapters written in these proceedings by E. Thiébaut, D. Mary, C. Aime.

I will conclude this section by giving some general considerations about image reconstruction with an interferometer. First of all, an interferometer made of $N$ telescopes produces $N(N-1) / 2$ baselines and thus samples $N(N-1) / 2$ frequencies in the Fourier transform of the brightness distribution of the object. We thus have a problem with $N(N-1)$ unknowns. 


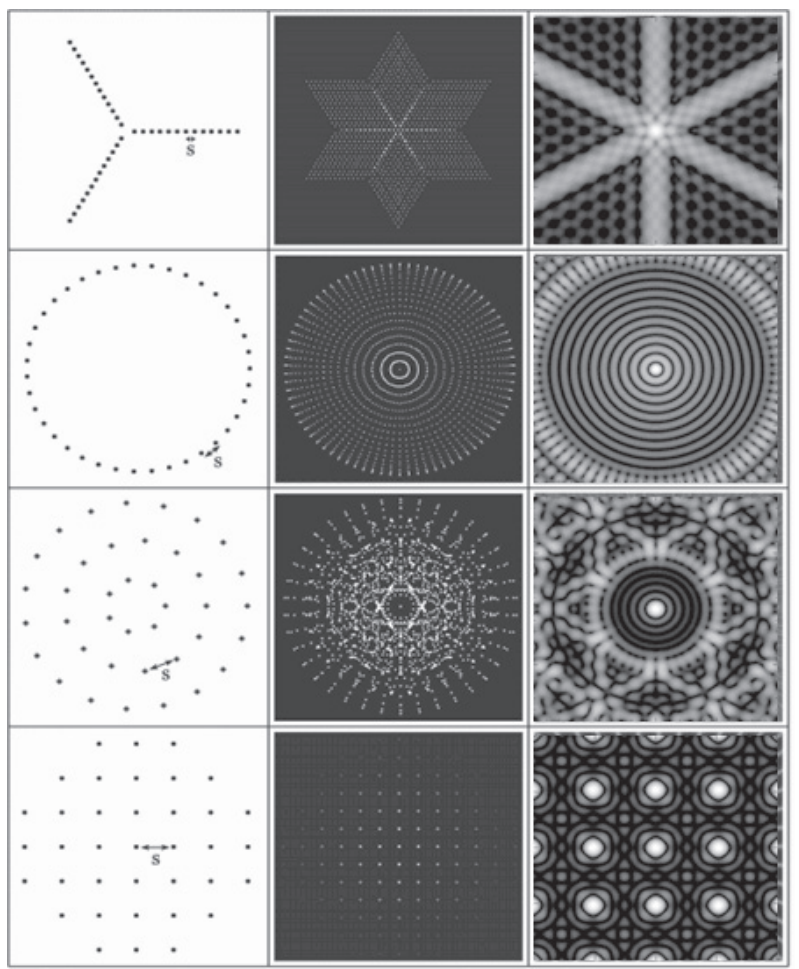

Fig. 2. Examples of $(\mathrm{u}, \mathrm{v})$ plane coverage (middle column) and of the corresponding point spread function (right column) for four different input pupil (left column) configurations.

We have already indicated that the limitations of ground based interferometers are dominated by the phase effects introduced by the atmospheric turbulence. If it is easy to measure the modulus of the Fourier transform over the $N(N-1) / 2$ points, the phase measurements are highly corrupted by the turbulence. As in radio interferometry, astronomers overcome this difficulty by computing closure phase measurements over triplets of apertures. It can be shown easily that the atmospheric phase terms are removed in the sum of the phase of three interference fringes over any triplet of telescopes. Thus closure phase measurements give us access to $(N-1)(N-2) / 2$ additional measurements. With this in hand, we understand that the problem is not well constrained because the number of unknowns is always larger than the number of measurements. A representation of these numbers is presented on Figure 3.

\section{Reality of optical interferometry}

The current implementation of optical interferometry involves a limited number of apertures. The VLTI is able to recombine four telescopes at the same time with the 


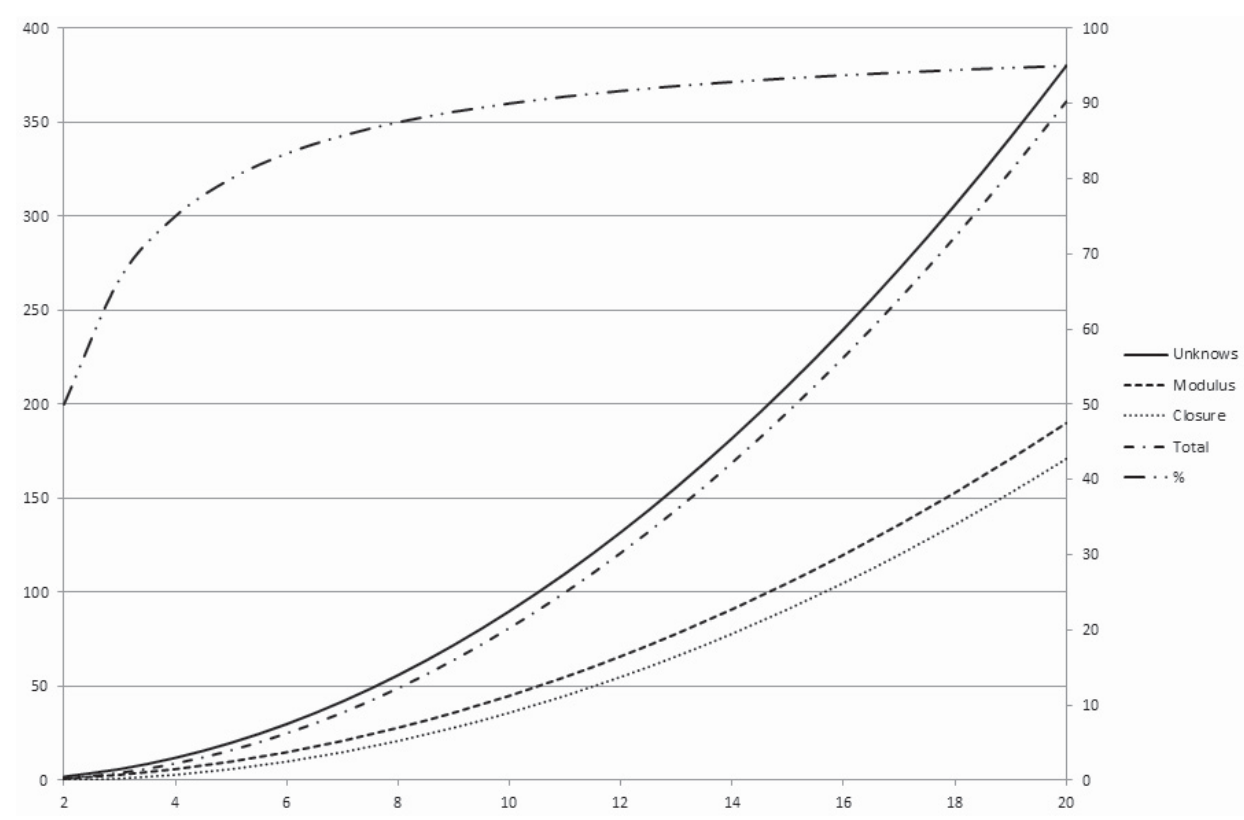

Fig. 3. Number of unknowns, of modulus and closure phase measurements as a function of the number of telescopes. The dark curve represents the percentage of information measured as a function of the number of telescopes.

PIONIER instrument (Le Bouquin et al. 2011) whereas CHARA can recombine up to 6 telescopes simultaneously with the MIRC instrument (Monnier et al. 2008). While this clearly corresponds to a great advance, it appears that imaging with optical interferometry is still very limited for the moment. Thus astronomers are mainly using the interferometric raw measurements (visibility, closure phase, complex differential visibility) to constrain the geometrical distribution of the emitting sources. An important effort has been devoted in the last years in the development of model fitting tools or of image reconstruction algorithms.

But before dealing with the calibrated products of the interferometer, many actions have to been done for a correct operation of the interferometer. One of the main difficulty concerns the overall reliability of the array. Indeed, an array is made of an important number of subsystems and the observing efficiency highly depends on the reliability of each element. This concerns the telescope (pointing, tracking, tip/tilt corrections, adaptive optics efficiency), the beam transportation and the optical path length equalization (vacuum pipes, optical fibers, optical quality, delay line, laser metrology, dumping of vibrations) and finally the beam combination and the signal sampling (spatial or temporal modulation, detection).

But on the ground and at optical wavelengths, the main difficulties for optical interferometry are in the domain of the perturbations induced by the atmospheric turbulence. The signal to noise ratio of a coherence measurement as described 
before depends of course on the coherence volume. This volume has in fact many dimensions. Spatial firstly and here the atmospheric turbulence highly reduces this dimension to the so-called Fried parameter $\left(r_{0}\right)$. Depending on the atmospheric conditions, the value of $r_{0}$ is in the range from 5 to $15-20 \mathrm{~cm}$, well below the diameter of the individual collectors. Temporal secondly and the reduction is here drastic with coherence time $t_{0}$ between 2 and $15 \mathrm{~ms}$ typically. Finally the spectral dimension should also be considered because the atmospheric turbulence has a spectral coherence characteristic that limit the useful spectral bandwidth to about 20 to $30 \mathrm{~nm}$ at visible wavelengths (Berio et al. 1997). Important efforts are devoted to improve this volume of coherence through the implementation of dilute adaptive optics systems: first adaptive optics to correct each aperture and then cophasing systems allowing to control the phase between the different sub apertures. With these conditions, long exposures will be possible and thus fainter magnitude and/or higher quality will be reached.

\section{The CHARA array and the VEGA spectro-interferometer}

The Center for High Angular resolution (CHARA) of the Georgia State University operates an optical interferometric array located at the Mount Wilson Observatory that consists of six one meter telescopes placed in pairs along the arms of a Y-shaped array. It yields 15 baselines ranging from 34 to $331 \mathrm{~m}$. Operating in the near-infrared with the instruments CLASSIC (Ten Brummelaar et al. 2005), CLIMB (Sturmann et al. 2010), FLUOR (Coude du Foresto et al. 2003), and MIRC (Monnier et al. 2008), and in the visible with PAVO (Ireland et al. 2008) and VEGA (Mourard et al. 2009, 2011), the CHARA array allows a maximum angular resolution of 1.3 and 0.3 millisecond of arc in the $\mathrm{K}$ and $\mathrm{V}$ band, respectively.

The VEGA spectrograph is designed to sample the visible band from 0.45 to $0.85 \mu \mathrm{m}$. It is equipped with two photon counting detectors looking at two different spectral bands. The main characteristics are summarized in Table 1. The optical design allows simultaneous recording of data, in medium spectral resolution, of the spectral region around $H \alpha$ with the red detector and around $H \beta$ with the blue detector. Observing in the blue requires good seeing conditions but increases by $30 \%$ the limit of spatial resolution of the instrument with respect to its operation around $700 \mathrm{~nm}$.

Table 1. Spectral resolution $(\mathrm{R})$ and bandwidth $(\Delta \lambda)$ of the VEGA spectrograph, as well as the spectral separation between the two detectors.

\begin{tabular}{rcrrr}
\hline Grating & R & $\Delta \lambda$ (Blue) & $\Delta \lambda$ (Red) & $\lambda_{R}-\lambda_{B}$ \\
\hline $\mathrm{R} 1: 1800 \mathrm{gr} / \mathrm{mm}$ & 30000 & $5 \mathrm{~nm}$ & $8 \mathrm{~nm}$ & $25 \mathrm{~nm}$ \\
$\mathrm{R} 2: 300 \mathrm{gr} / \mathrm{mm}$ & 5000 & $30 \mathrm{~nm}$ & $45 \mathrm{~nm}$ & $170 \mathrm{~nm}$ \\
\hline
\end{tabular}

The limiting magnitudes of VEGA/CHARA are presented in Table 2. They of course highly depend on the actual seeing conditions and on the intrinsic target visibility. 
Table 2. Estimation of typical limiting magnitude as a function of the different spectral resolution modes. These values are presented for the median value of the Fried parameter $r_{0}$ at Mount Wilson i.e. $8 \mathrm{~cm}$. We also indicate the best performances assuming an $\mathrm{r} 0$ of $15 \mathrm{~cm}$.

\begin{tabular}{|c|c|c|c|}
\hline Resolution & $\mathrm{R}$ & Typical Lim. Magnitude & Best perf. \\
\hline Medium & 6000 & 6.5 & 7.5 \\
High & 30000 & 4.2 & 5.5 \\
\hline
\end{tabular}

VEGA is in routine operation at Mount Wilson and benefits from about 60 nights per year. Many observations are now done remotely from Nice Observatory. Our group has recently improved the photon counting detectors. A new image intensifier has been installed with better quantum efficiency (approximately a factor 1.5 better) in the red part of the spectrum and the Dalsa sensor (Blazit et al. 2008) behind the two image intensifiers has been replaced by a Gazelle sensor from the Point Grey company. This new sensor allows a faster frame rate $(10 \mathrm{~ms})$ and a much lower dead time during two frames $(1 \mathrm{~ms}$ instead of $2 \mathrm{~ms}$ ). The duty cycle of the sensor is now of the order of $90 \%$ instead of $60 \%$ with the old camera. An improvement of 1.5 magnitude has thus been recently demonstrated as well as a much better detector cosmetics important for spectrum measurements.

We are also considering a future evolution of VEGA in order to correctly benefit from the future installation of adaptive optics on the CHARA telescopes. The high Strehl ratio that will be allowed thanks to these new devices will highly increase the signal to noise of our measurements. However it will also concentrate the flux in a small part of the detector and thus will lead to an increase of the saturation effect with the current generation of photon counting detector. We are thus considering using analogical detector such as EMCCD or OCAM2 (Feautrier et al. 2011) that allows a very high frame rate (up to $1500 \mathrm{fps}$ ) and a very low readout noise $\left(0.13 e^{-} / \mathrm{pix} /\right.$ frame $)$. Coupling this kind of detector with a beam combiner using spatial filtering and high efficiency optical devices (P. Bério, in preparation) will permit to enhance the scientific domain of VEGA/CHARA in the future.

\section{Recent results from VEGA/CHARA}

The most remarkable properties of VEGA/CHARA are first the access to unprecedented angular resolution thanks to the 300 meters baseline and the short wavelengths and second the access to high angular resolution measurements at very high spectral resolution (up to 30 000).

The medium (6000) and high (30 000) spectral resolutions are well suited to perform kinematic analysis of the interferometric signal, providing resolution of 60 and $10 \mathrm{~km} \mathrm{~s}^{-1}$ respectively. These spectral resolutions are best dedicated to the extraction of differential spectral information. Radiative winds and fast rotating 
photospheres of hot stars can be probed efficiently with the medium spectral resolution. Some recent examples of such studies could be found for the Be stars 48 Per and $\phi$ Per (Delaa et al. 2011) where the authors characterize the rotating disks in term of extension, ellipticity and kinematical field. In Meilland et al. (2011), the authors use the combination of VEGA and VLTI/AMBER (Petrov et al. 2007) data to constrain both the orbital elements of the famous Be binary $\delta$ Sco and the disk's parameters. The interactive binaries $\beta$ Lyrae and $v$ Sagitarri (Bonneau et al. 2011) have also been studied in the same way. Perraut et al. (2010) succeeded also for the first time to spectrally and spatially resolve the $H \alpha$ emitting region of the prototype of the young stellar objects AB Aurigae. High spectral and angular resolutions bring also complementary views on old and famous problems such as the mysterious eclipsing system $\epsilon$ Aurigae (Mourard et al. 2012) or on the chromosphere of $\mathrm{K}$ giants (Berio et al. 2011).

The medium resolution is also well suited to absolute visibility studies and are also well adapted for the study of binaries or multiple systems. In that field the main goal is the study of fundamental stellar parameters through angular diameters measurements and analysis through classical stellar modeling and/or confrontation with other observing techniques such as spectroscopy and asteroseismology. Recent results of such programs concern the study of the ro Ap star $\gamma$ Equulei (Perraut et al. 2011) or the famous CoRoT targets HD49933 (Bigot et al. 2011) and more recently the study of four exoplanet hosts stars (Ligi et al. 2012). These exploratory programs are now coordinated as large programs where many tens of objects are being studied in order to have a good analysis of the stellar properties in different part of the Herztsprung-Russel diagram.

Another interesting possibility is the presence of a polarimeter that could be inserted into the beam. This gives new insight into many physical processes. Many science sources are linearly polarized, in particular at a small angular scale, and the interferometric polarized signal is a powerful probe of circumstellar scattering environments that contain ionized gas or dust (Chesneau et al. 2003; Elias et al. 2008; Ireland et al. 2005) and of magnetic properties (Rousselet-Perraut et al. 2000, 2004). This possibility has not yet been really exploited on the VEGA/CHARA interferometer but it could bring interesting new programs.

\section{Conclusion}

With this lecture and this paper, my intention has been first to describe the way optical interferometry should be understood from a physical point of view and second to show the recent advances in that field in terms of astrophysical programs and in terms of observing possibilities. The dream of the groups working in optical interferometry is clearly to push towards a large facility with remarkable capabilities highly complementary to what will bring, in the future, the Extremely Large Telescopes or the large radio arrays.

In complement to the science addressed by the large radio arrays and the Extremely Large telescopes, we consider that optical interferometry can bring important answers, firstly on the possibility of fighting off the expected confusion 
limit of ELT and secondly for the direct imaging with spatial, temporal and spectral resolution of compact sources such as the inner part of young stellar objects where planets are formed or the inner parsec around active galactic nuclei. In all cases, the quality of the synthetic point spread function will be fundamental both for the sensitivity and for the resolving power. The control of such imaging machines for nulling or phase-controlled coronagraphy is also of utmost importance for the detection and characterization of planets in the habitable zone. In this latter case, the effort is more in the control of the dynamic in the image than in the angular resolution. Debates around the future concepts have almost concluded around three main classes of future optical arrays: 1) a VLTI-like interferometer with a very small number of ELT-like telescopes on a compact array, 2) kilometric baselines with a small number of 8-m class telescopes and 3) a dense array of a large number of small telescopes over possibly kilometric baselines. If the conceptual design of the two first classes of array could certainly rest on the current concepts of classical telescopes + delay lines, it is clear that expanding the number of apertures to 50, 100 or even more individual apertures encounters a real limitation for the implementation. This represents a major difference to the situation of radio interferometry and many conceptual and prototyping efforts are now engaged in that direction.

\section{References}

Berio, P., Mourard, D., Vakili, F., et al., 1997, JOSA-A, 14

Berio, P., Merle, T., Thevenin, F., et al., 2011, A\&A, 535

Bigot, L., Mourard, D., Berio, P., et al., 2011, A\&A, 534

Blazit, A., Rondeau, X., Thiebaut, E., et al., 2008, Appl. Opt., 47

Bonneau, D., Chesneau, O., Mourard, D., et al., 2011, A\&A, 532

Chesneau, O., Wolf, S., \& Domiciano de Souza, A., 2003, A\&A, 410

Colavita, M., Serabyn, G., Wizinowich, P., \& Akeson, R., 2006, Proc. SPIE, 6268

Coude du Foresto, V., Borde, P., Merand, A., et al., 2003, SPIE Conf. Proc., 4838

Delaa, O., Stee, P., Meilland, A., et al., 2011, A\&A, 529

Elias, N., Schmitt, H., Jorgensen, A., et al., 2008 [arXiv:0811.3139]

Feautrier, P., Gach, J.L., Balard, P., et al., 2011, PASP, 123

Glindemann, A., et al., 2004, Spie Proc., 5491

Goodman, J.W., 2000, "Statistical Optics" (Wiley)

Haubois, et al., 2008, A\&A

Ireland, M., Tuthill, P., Davis, J., \& Tango, W., 2005, MNRAS, 361

Ireland, M., ten Brummelaar, T., Tuthill, P.G., et al., 2008, SPIE Conf. Proc., 7013

Labeyrie, A., 1975, ApJ, 196

Labeyrie, A., 1996, A\&AS, 118

Lacour, et al., 2008, A\&A

Le Bouquin, J.B., Berger, J.P., Lazareff, B., et al., 2011, A\&A, 535

Ligi, R., Mourard, D., Lagrange, A.M., et al., 2012, A\&A, 545 
Meilland, A., Delaa, O., Stee, P., et al., 2011, A\&A, 532

Monnier, et al., 2007, Science

Monnier, J., Zhao, M., Pedretti, E., et al., 2008, SPIE Conf. Proc., 7013

Mourard, D., Clausse, J.M., Marcotto, A., et al., 2009, A\&A, 508

Mourard, D., Berio, P., Perraut, K., et al., 2011, A\&A, 531

Mourard, D., Harmanec, P., Stencel, R., et al., 2012, A\&A, 544

Perraut, K, Benisty, M., Mourard, D., et al., 2010, A\&A, 516

Perraut, K., Brandao, I., Mourard, D., et al., 2011, A\&A, 526

Petrov, R., Malbet, F., Weigelt, G., et al., 2007, A\&A, 464

Rousselet-Perraut, K., Chesneau, O., Berio, P., \& Vakili, F., 2000, A\&A, 354

Rousselet-Perraut, K., Stehle, C., Lanz, T., et al., 2004, A\&A, 422

Sturmann, J., ten Brummelaar, T., Sturmann, L., \& Mc Alister, H.A., 2010, SPIE Conf. Proc., 7734

ten Brummelaar, T., McAlister, H.A., Ridgway, S., et al., 2005, ApJ, 628 